\title{
DIFFUSE LEED INTENSITIES OF DISORDERED CRYSTAL SURFACES
}

\author{
III. LEED investigation of the disordered (110) surface of gold \\ D. WOLF, H. JAGODZINSKI and W. MORITZ \\ Institut für Kristallographie und Mineralogie der Universität München, Theresienstrasse 41, \\ D-8000 München 2, Germany
}

Received 5 August 1978; manuscript received in final form 3 April 1978

The LEED pattern of clean (101) surfaces of $A u$ show a characteristic $(1 \times 2)$ superstructure. The diffuseness of reflections in the reciprocal [010] direction is caused by onedimensional disorder of chains, strictly ordered into spatial [101] direction. There is a transition from this disordered superstructure to the normal $(1 \times 1)$ structure at $420 \pm 15^{\circ} \mathrm{C}$. The angular profiles of the $\left(0 \frac{1}{2}\right)$ and (01) beam are measured at various temperatures and with constant energy and angles of incidence of the primary beam. The beam profiles are deconvoluted approximately with the instrument response function.

\section{Introduction}

The development of dynamical theories for the diffraction of low energy electrons [1] is based on the assumption of a geometrically perfect crystal lattice of the surface with rigid periodicity in two directions; only the components of the atomic parameters, normal to the surface, may vary. However, a two-dimensional undisturbed arrangement of the surface atoms of real crystals is rarely observed. In general, the defects of the surface structure due to the large amplitudes of thermal vibrations cannot be neglected, i.e., real surfaces are disordered. In diffraction experiments with low energy electrons the influence of disorder on the intensity distribution of the scattered electrons can be recognized frequently in spite of the relatively low resolution of the conventional LEED diffractometers. An angular displacement of beam positions or a clear diffuseness of beams in the different crystallographic directions can be observed.

According to the results of various LEED calculations and experiments an undisturbed surface lattice necessarily required an accurate dynamical theory for the solution of the diffraction problem. For disordered crystals however, the exact solution of the multiple scattering problem, as developed in parts I and II [2], is much more complicated. Therefore, the order parameters only may be determined by an analysis of the angular beam profiles under certain conditions, that means, the 
angular profile of a reflection at constant energy is evaluated only. Compared with the usual procedure of determination of the structure parameters the determination of order parameters becomes facilitated.

According to the theoretical treatment given in parts I and II the LEED investigation of disordered surface structures requires the solution of a two-dimensional disorder problem, which may often be described in terms of a one-dimensional model of disorder. Here the statistical distribution of faults involves one strictly ordered direction of the surface lattice only, corresponding to diffuse streaks in the diffraction pattern. The statistics of this special case can be solved by a simple matrix formalism. The diffraction apparatus and the experimental results are described in this article. In part IV a special structure model of the disordered (101) gold surface is given, and its diffraction patterns are interpreted in terms of this model.

\section{Experimental equipment and techniques}

\subsection{The diffraction apparatus}

The ultra-high vacuum system, the bakeable diffraction chamber of stainless steel, and the LEED diffractometers with energy analyzing Faraday cups, the ion gun, and the special precision manipulators for the crystal and gun adjustment are our own developments and described elsewhere [3]. The pumping system consists of a mechanical forepump, an oil diffusion pump and a sputter pump. Molecular sieve traps and liquid nitrogen cold trap baffles in the exhaust tubes suppress backstreaming of pumping fluid to the ultra high vacuum system. Analyses of the composition of residual gas in the diffraction chamber show for hydrocarbons partial pressures below $7 \times 10^{-10} \mathrm{~Pa}$; the normal pressure runs up to about $5 \times$ $10^{-8} \mathrm{~Pa}$ during the continuous running of the electron optics' assemblies and the specimen heating. By means of a bakeable manifold auxiliary gas inlet the partial pressure of a special gas, or a mixture of gases, can be admitted or varied. The gas composition in the diffraction chamber is controlled by a magnetic residual gas analyser. For cleaning the gold surfaces by ion bombardment with argon, or other inert gas ions, a special gun with an electrostatically focussed ion beam, variable in intensity and energy, has been set up.

\subsection{Electron optics assemblies}

During our investigations of the (101)Au surfaces we used three different LEED diffractometers:

(a) For general views and for the determination of the thermal stability range of the surface structures we had a conventional two-grid backscattering system with an angle of acceptance from the target at $120^{\circ}$. 
(b) For the measurement of energy distribution function of the scattered electrons and for Auger electron spectroscopy we used a modified four-grid LEED system $[3,4]$. The usual electronic equipment for the energy analysis was completed by an additional Schering bridge for capacitance compensation of the retarding field analyser and by a band pass amplifier to suppress the fundamental frequency [5].

(c) The investigations of the disordered gold surfaces were done with a universal LEED diffractometer $[3,6]$.

The principle of these LEED optics is represented in fig. 1. The small electron gun is arranged in front of the fluorescent screen and can be rotated through $360^{\circ}$ within the equatorial plane; the angle of incidence for the primary electrons is freely adjustable between 0 and $90^{\circ}$. Thus, the intensity of all reciprocal surface lattice points can be measured either by a variation of the primary energy or by variation of the angle of incidence. The advantage of the conventional backscattering LEED system, i.e., the visibility of the diffraction pattern, is preserved by a two-grid fluorescent screen assembly with the angles of acceptance from the target of $140^{\circ}$ for the azimuth and $\pm 25^{\circ}$ for the vertical circle [7].

A pair of deflecting plates between the crystal and the fluorescent screen allows for a vertical compression of the whole diffraction pattern or may shift it such that beams lying outside the screen can be observed too.

The scattered intensity can be measured with three energy analysing Faraday cups. Two of them are positioned stationary behind holes in the screen; their diameters are $5 \mathrm{~mm}$ and $1 \mathrm{~mm}$ respectively; the third cup's entrance aperture diameter of $1.2 \mathrm{~mm}$ is next to the innermost grid of the LEED optics and can be moved on spherical coordinates to every place of the screen. The mechanical adjustment of

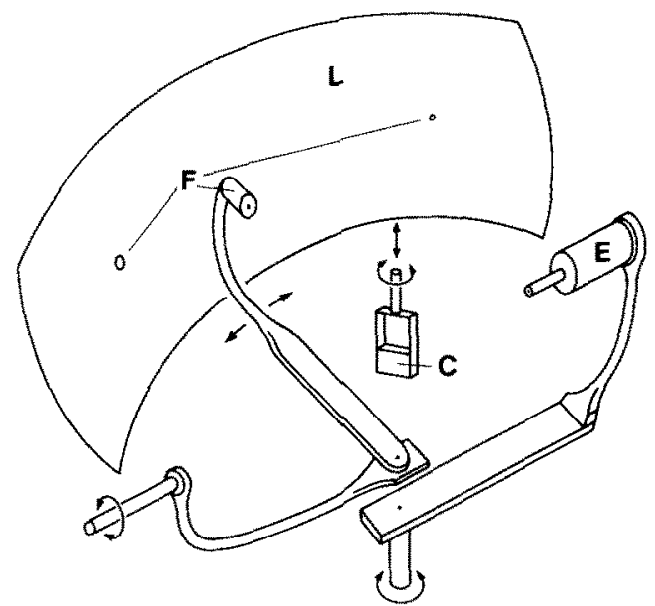

Fig. 1. Schematic drawing of the LEED assembly: E, movable electron gun; C, crystal; F, Faraday cups; L, LEED screen with two grids. 
the specimen, the electron gun and the Faraday cage, are done with special precision manipulators [3]; the adjustment precision of the manipulator amounts to $0.01^{\circ}$ at unidirectional motion, respectively $0.1^{\circ}$ at a return of movement.

\subsection{Target preparation and cleaning}

Three different gold crystals with (101) surfaces have been prepared for these diffraction experiments. Their diffraction patterns were practically identical.

The commercial gold crystals of $6 \mathrm{~N}$ purity were first oriented by using X-ray diffraction methods, in order to guarantee surfaces normal to the exact crystallographic [101] direction within $0.5^{\circ}$. Then the crystals were cut by spark erosion. The targets were electrolytically etched in $5 \mathrm{n}_{2} \mathrm{SO}_{4}$, and finally electropolished in a cyanide solution. These crystals showed smooth reflecting surfaces. The target cleaning procedures in the LEED chamber consisted of low energy argon ion sputtering, outgassing and annealing under ultra-high vacuum conditions at $250^{\circ} \mathrm{C}$ to remove bombardment damages. Annealing temperatures below $200^{\circ} \mathrm{C}$ caused no visible sharpening of the beam profiles. The dependence on the annealing time of the profile width of a normal and a superstructure beam at $50 \mathrm{eV}$ primary energy is shown in fig. 2 . The tempering procedure was finished when the profile measurement showed constant full width at half maximum of the beam.

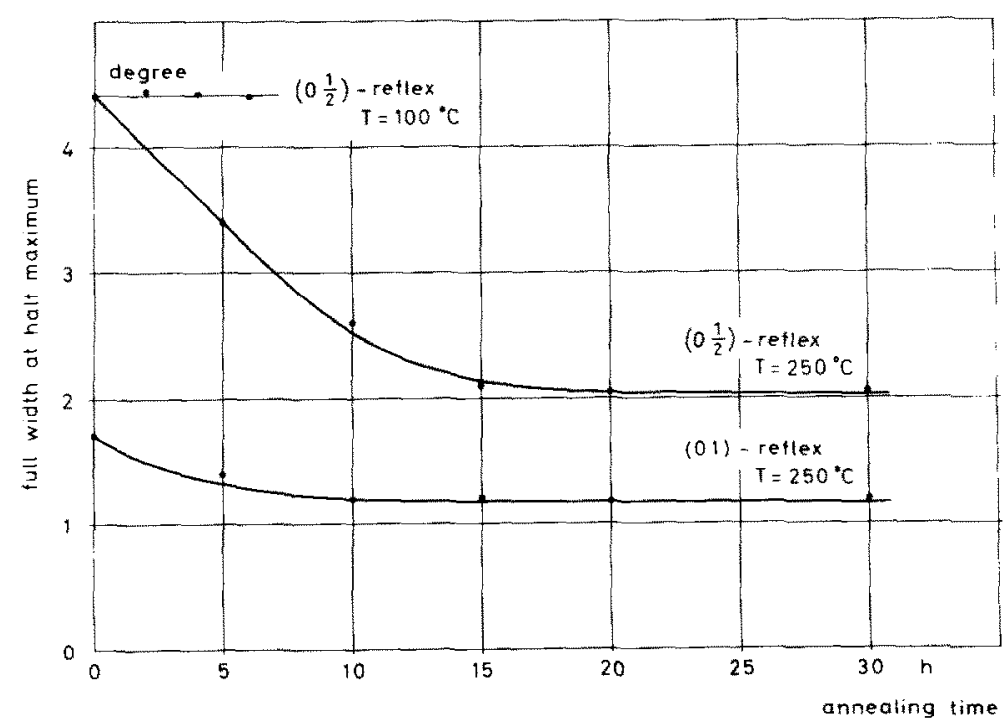

Fig. 2. Full width at half maximum of the reflexes versus annealing time after ion bombardment with $280 \mathrm{eV}$ argon ions and ion density of $300 \mu \mathrm{A} \mathrm{cm}^{-2}$, during $10 \mathrm{~min}$. 


\section{Experimental results}

\subsection{Diffraction patterns}

A $(1 \times 2)$ structure with a doubled lattice constant in [010] direction was found to be characteristic for the (101)Au surface. The diffraction pattern (figs. 3a to 3f) shows, besides the normal beams with Miller indices $(h, k)$, additional half order beam patterns in the $[010]^{*}$ direction of the reciprocal lattice. Normal $(1 \times 1)$ bulk-type diffraction patterns of low intensity only were observed immediately after electropolishing and under high vacuum conditions. They were extremely unstable and disappeared after heating the crystal up to $300^{\circ} \mathrm{C}$. In spite of a systematic variation of the special ion bombardment and annealing parameters we couldn't produce a stable $(1 \times 1)$ bulk-type pattern after exposure of the crystal surface to ultra-high vacuum. Auger electron spcctroscopy of the surfaces showed no contamination by adsorbed atoms. Therefore the $(1 \times 2)$ superstructure scems to be typical for a stable arrangement of atoms of the clean (101)Au surface.

The distincl bruadening of both nomial beams and superstructure beams, which is dependent on the primary electron energy, can be attributed to the statistical faults of the superperiodicity, consequently the (101)Au surface with the $(1 \times 2)$ superstructure is disordered. As beam broadening just appears in one direction of the reciprocal lattice, a one-dimensional disorder problem is realized. It is evident that with increasing primary electron energy all beams become sharper (figs. 3e, 3f); above $150 \mathrm{eV}$ no clear differences in the width of bulk-type and superstructure beams can be observed. At a greater depth of penetration of the electrons the beam profile is mainly determined by the strong periodicity of the bulk crystal. Then the influence of the disordered surface area of a thickness of one to two atom layers upon the intensity profile is less significant, although the superstructure reflections are still visible.

In order to get LEED profiles of high accuracy, the diffracted beams were measured only after having checked the temperature stability of the (101)Au surface structure.

The thermal range of stability of the $(1 \times 2)$ superstructure has been determined by annealing experiments (annealing time 1 to $50 \mathrm{~h}$ ) with a systematic variation of the crystal temperature between 20 and $800^{\circ} \mathrm{C}$.

Photographic LEED patterns could be taken up to a crystal temperature of $450^{\circ} \mathrm{C}$; intensity profile measurements with the Faraday collectors were still possible at $600^{\circ} \mathrm{C}$. The influence of temperatures above $600^{\circ} \mathrm{C}$ upon the surface structure could only be examined indirectly, i.e., after cooling the crystal to room temperature.

The $(1 \times 2)$ superstructure is visible up to a surface temperature of $400^{\circ} \mathrm{C}$. Above $380^{\circ} \mathrm{C}$ the intensity of the fractional order reflections diminished rapidly; at $420^{\circ} \mathrm{C}$ the diffraction patterns (figs. $4 \mathrm{a}-4 \mathrm{~d}$ ) show only normal lattice reflections.

In intensity measurements with the Faraday cup the superstructure beams are 

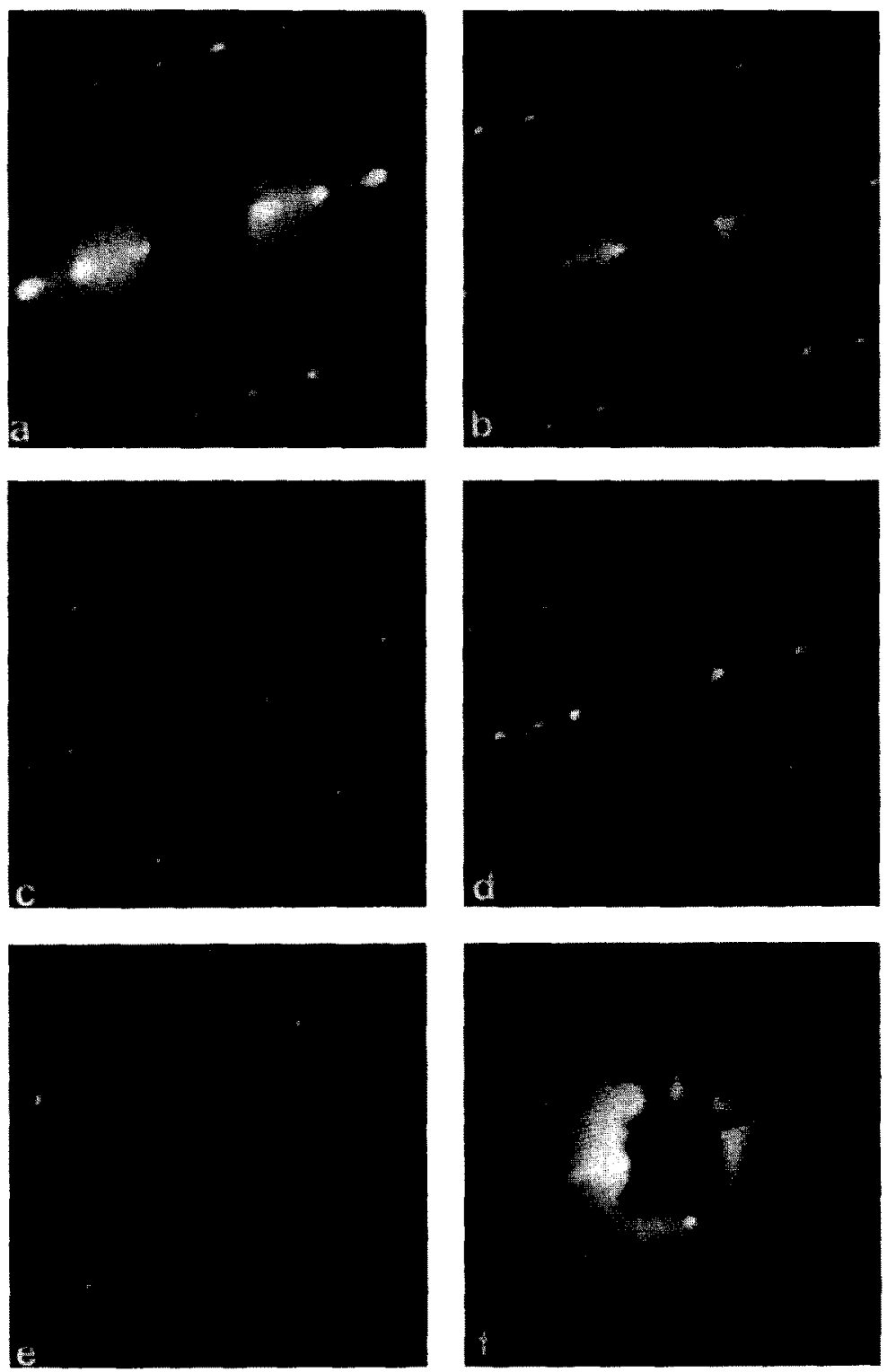

Fig. 3. Backscattering patterns of the (101) Au surface with $(1 \times 2)$ superstructure: (a) $50 \mathrm{eV}$; (b) $70 \mathrm{eV}$. (c) $87.5 \mathrm{eV}$; (d) $125 \mathrm{eV}$; (e) $160 \mathrm{eV}$; (f) $240 \mathrm{eV}$

detectable up to $515^{\circ} \mathrm{C}$, the more intense integer order beams begin to disappear in the incoherent background from $600^{\circ} \mathrm{C}$ upwards. After cooling the crystal below the critical temperature the superstructure reflections appear again, i.e., the struc- 

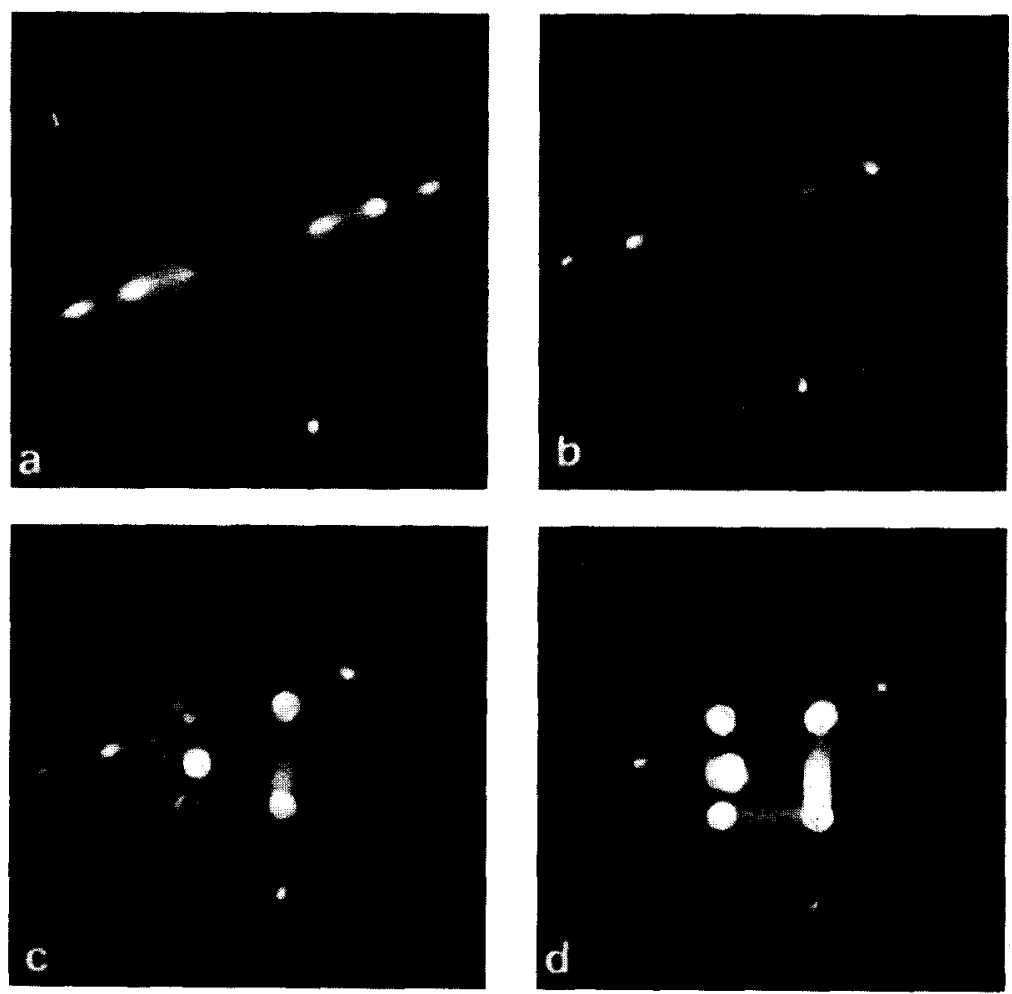

Fig. 4. Diffraction patterns of the (101)Au surface during annealing of the crystal. Electron energy $E_{\mathrm{p}}=50 \mathrm{eV}$. (a) $25^{\circ} \mathrm{C}$; (b) $300^{\circ} \mathrm{C}$; (c) $350^{\circ} \mathrm{C}$; (d) $400^{\circ} \mathrm{C}$.

ture transformation of the (101) Au surface,

$(1 \times 2) \stackrel{400-450^{\circ} \mathrm{C}}{=}(1 \times 1)$,

is reversible.

After annealing under UHV condition, $p<5 \times 10^{-8} \mathrm{~Pa}$, for more than $3 \mathrm{~h}$ and with temperatures above $500^{\circ} \mathrm{C}$, another type of superstructure was observed. Besides the $(1 \times 2)$ superstructure beams the diffraction pattern contains additional fractional order beams on hexagonal positions [3]; in analogy to the superstructure of the $(100) \mathrm{Au}$ surface $[8,9]$, this structure can be explained by a hexagonal arrangement of atoms with a lattice constant of $2.88 \AA$ in the uppermost atom layer*. Nevertheless, this hexagonal surface structure is not important for the disorder under discussion.

* A hexagonal structure with a lattice constant differing only slightly from the bulk lattice was also detected on the (111) surface of gold after long-time annealing [3]. 
The present study of the disordered (101) gold surface has been restricted to the clearly defined thermal stability range of the $(1 \times 2)$ superstructure.

\subsection{Measurements of the intensity profiles}

A specific aim of this study was to measure the angular beam profiles in order to extract the statistical parameters by a direct analysis of the experimental data. For this purpose the angular intensity profiles of the $(01)$ and the $\left(0 \frac{1}{2}\right)$ beams have been registered.

The experimental equipment with LEED gun, display system, and Faraday cup, is schematically illustrated in fig. 5. The reciprocal lattice and the Ewald sphere for the corresponding parameters which are kept constant for all profile measurements (primary energy $E_{\mathrm{p}}=68 \mathrm{eV}$ and angle of incidence $\vartheta_{0}=47^{\circ}$ ) are shown in fig. 6 .

These special diffraction parameters take into consideration the shape of curve of the atomic scattering factor $f(\vartheta)$, which is approximately constant within the range of a scattering angle of $80-100^{\circ}$ [10]. By multiple scattering, of course, the effective scattering amplitude might show a much stronger angular dependence. However, this could not be observed experimentally as the variation of the angular incidence did not cause a rapid variation of the diffracted intensities.

The profile measurements were done by moving the Faraday cup along the $[010]^{*}$ direction of the diffraction pattern until constant background intensity had been reached on both sides of the interference maximum.

The linear movement of the collector was connected with the X-scale of an

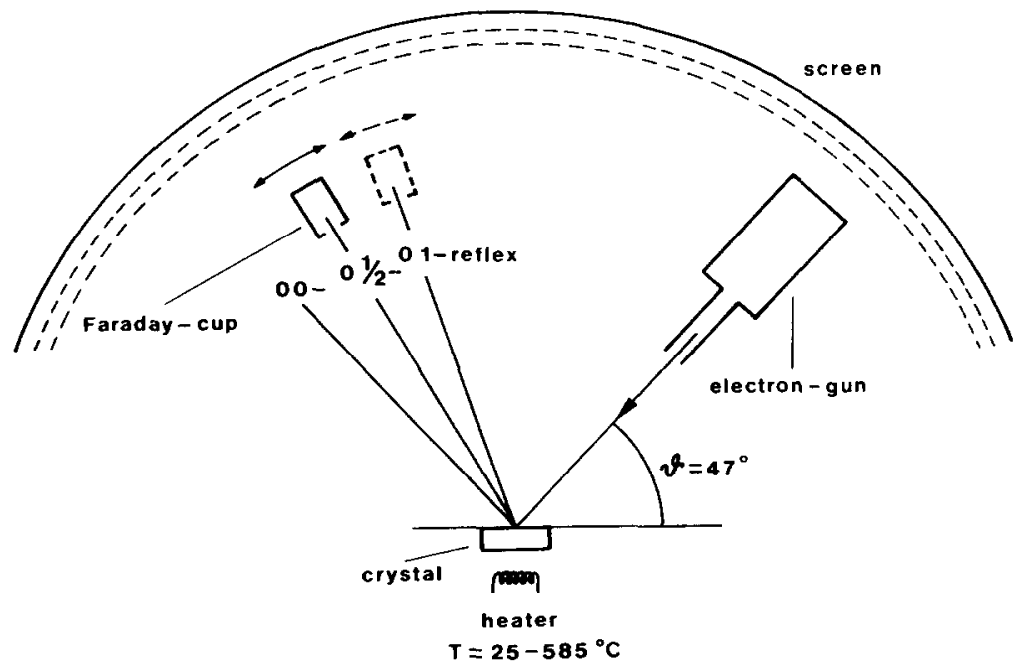

Fig. 5. Geometrical arrangement of electron gun and Faraday cup for measurements of beam profiles. 


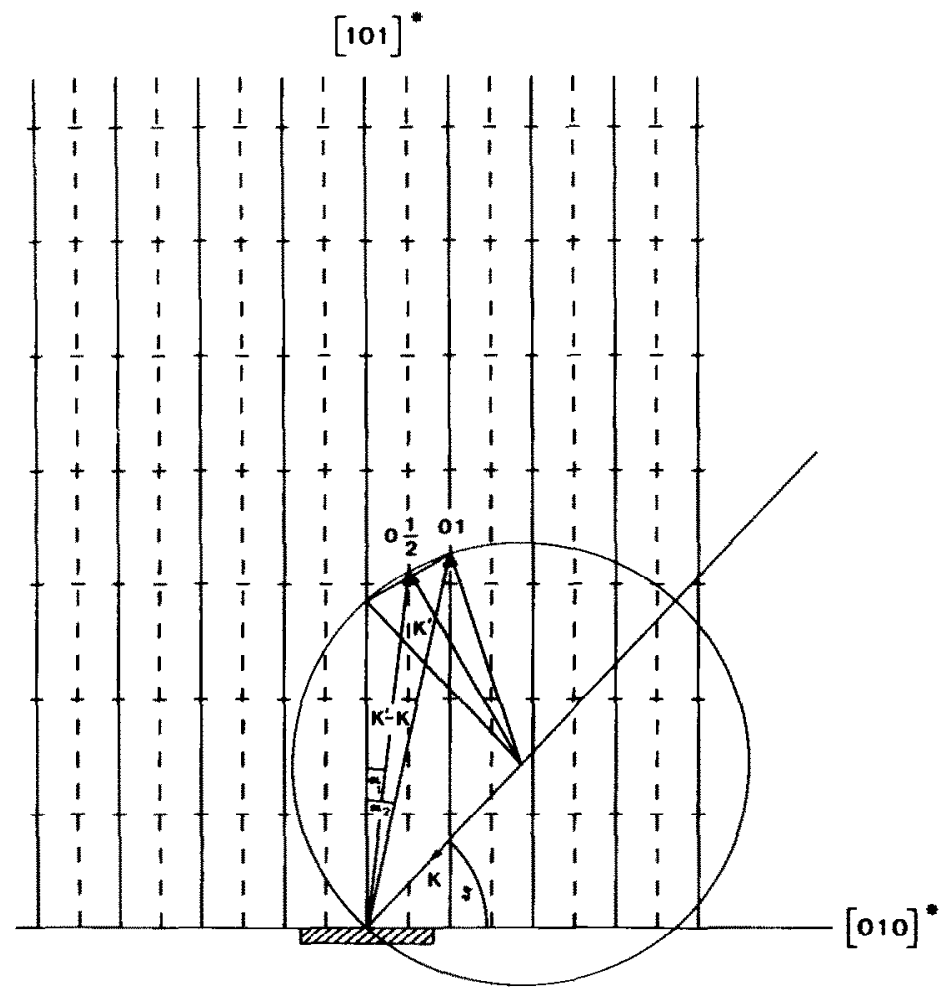

Fig. 6. $(0 k l)$ intersection of the reciprocal lattice of the (101)Au surface with $(1 \times 2)$ superstructure and Ewald sphere. Full lines: lattice rods for normal reflections; dashed lines: lattice rods for superstructure reflections; cross lines indicate the kinematical reflex positions on the rods.

$X-Y$ plotter, the $Y$ coordinate recorded the intensity. This method produced directly the intensity profile; the integral intensities were determined by a graphic integration of these curves.

Inelastic scattered electrons were suppressed by a retarding field in front of the collector; the specific internal construction of the Faraday cup [3], a collector potential about $50 \mathrm{eV}$ above the primary energy level, and potential guarded screened cables, ensure that the intensity measurements are free from secondary electrons or from leakage currents as a result of too low isolation resistances.

The shape of the intensity profiles as a function of the surface temperature was examined within the temperature range of $25^{\circ} \mathrm{C}$ to $585^{\circ} \mathrm{C}$. The temperature was measured with a Pt-PtRh thermocouple, fixed to the crystal surface.

With increasing temperature both the maximum of the beams and the integral intensity become smaller, while the full width at half maximum increases; fig. 7 shows typical beam profiles at different temperatures. Within the temperature range 

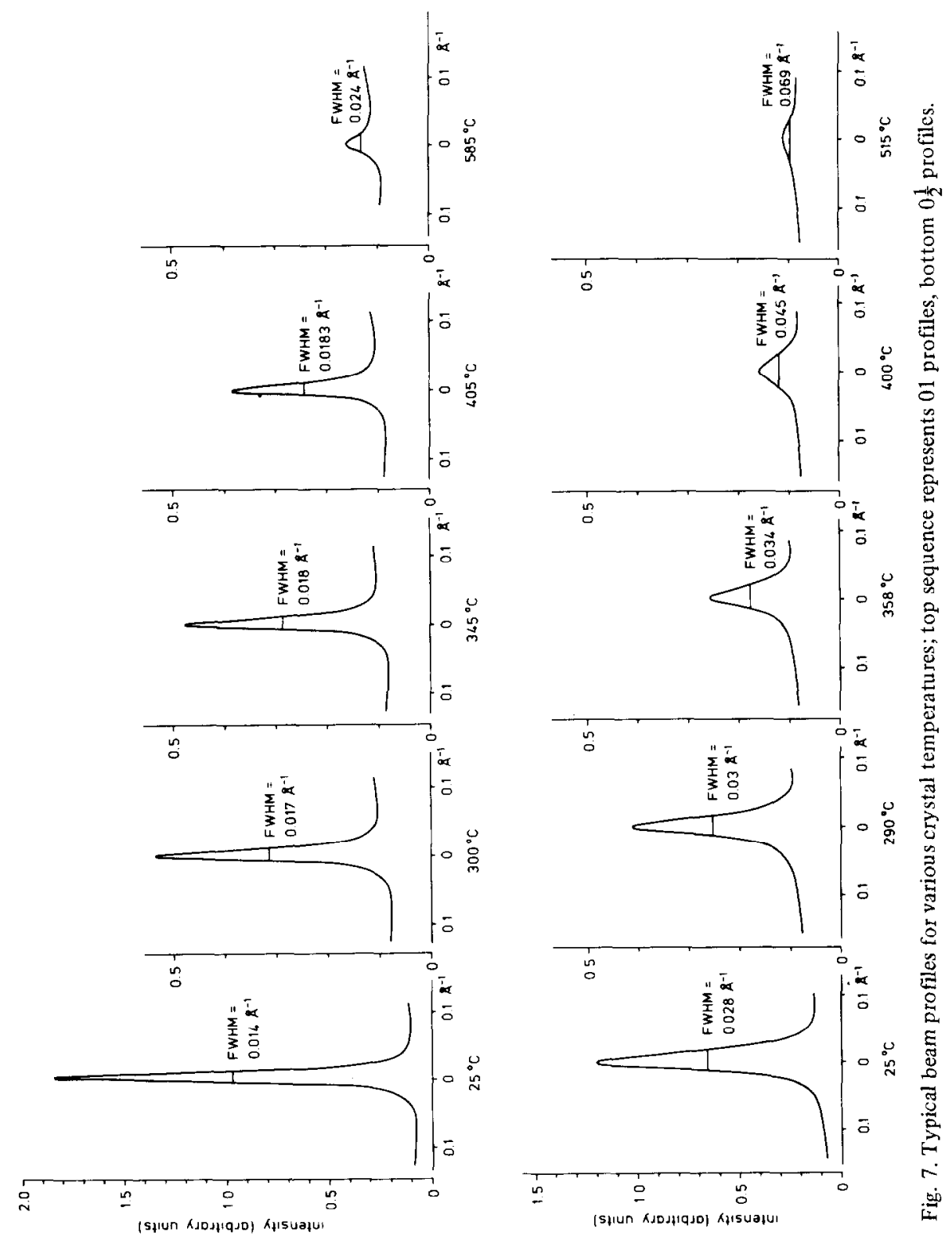


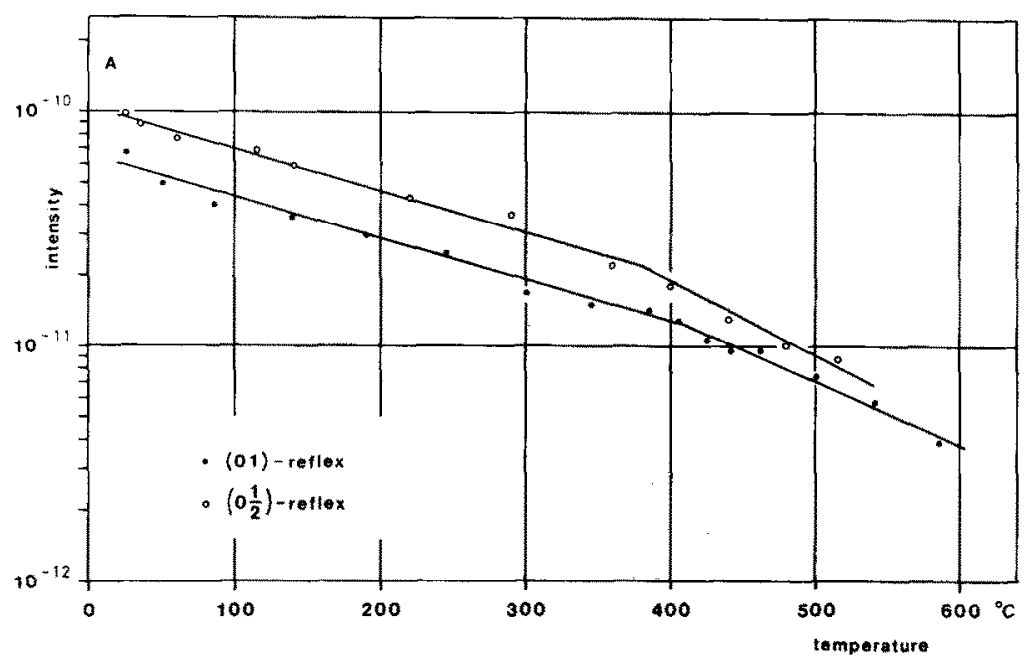

Fig. 8. Integral intensity of the (01) and $\left(0 \frac{1}{2}\right)$ beams as a function of temperature.

of 25 to about $400^{\circ} \mathrm{C}$ the functional dependence of the intensity of the $(01)$ beam as well as of the superstructure beam is given by an exponential function or in a logarithmic plot by straight lines of equal slopes; fig. 8 shows a plot of $\log I$ versus the temperature $T$. Between 380 and $400^{\circ} \mathrm{C}$ these straight lines bend down; the slope becomes steeper and is now different for the two reflexes. This bend in the intensity curve $I(T)$ is correlated with the vanishing of the superstructure reflexes in the diffraction diagram.

For crystal temperatures above $585^{\circ} \mathrm{C}$, respectively $515^{\circ} \mathrm{C}$, the measurements of the $(01)$ and $\left(0 \frac{1}{2}\right)$ beam profile are inaccurate, because of the low hight of the interference (fig. 7) and the corresponding width of the reflection.

\section{Evaluation of intensity measurements}

The beam profile measurements cannot be analyzed directly. The required disorder parameters, such as the probabilities for distortions of the lattice periodicity, can be determined with sufficient accuracy only from the intrinsic profile. The observed profile is the convolution product of the various individual instrumental profiles and the intrinsic interference function. The latter includes all effects caused by the disordered crystal surface. As a consequence of this convolution all observed profiles are broadened.

The determination of the true intensity profile of a reflected beam requires a deconvolution with a single instrumental function which combines the different instrumental component profiles. From the deconvoluted beam profiles all disorder parameters can be derived in a first approximation. As this investigation is a first 
application of the disorder theory on surface structure analysis no exact deconvolution procedure has been used here for the purpose of simplicity. The instrumental profile has been eliminated by a simple method of approximation, which is less sophisticated and requires a minimum of computing time only.

For some individual profiles an exact numerical deconvolution method has been used to prove the validity of the approximation described below. The differences of the profiles deconvoluted by both methods are minimal and can be neglected.

First of all, the analytical shapes of the instrumental function and the observed beam profiles have been determined. The instrumental profile is represented by the convolution product of the primary beam profile, including thermal energy distribution, with the detector function. All observed profiles include this convolution product, therefore, an additional unfolding of the instrumental function is unnecessary. This instrumental profile has been determined directly with the Faraday cup as a function of the primary energy of the electrons. These measurements have been done with the direct beam.

The shape of the instrumental profile (fig. 9) satisfies in a good approximation the Gaussian function

$y=a \exp \left(-h^{2} x^{2}\right)$;

only the wings are slightly different.

This instrumental profile can be applied directly to unfold the observed intensity

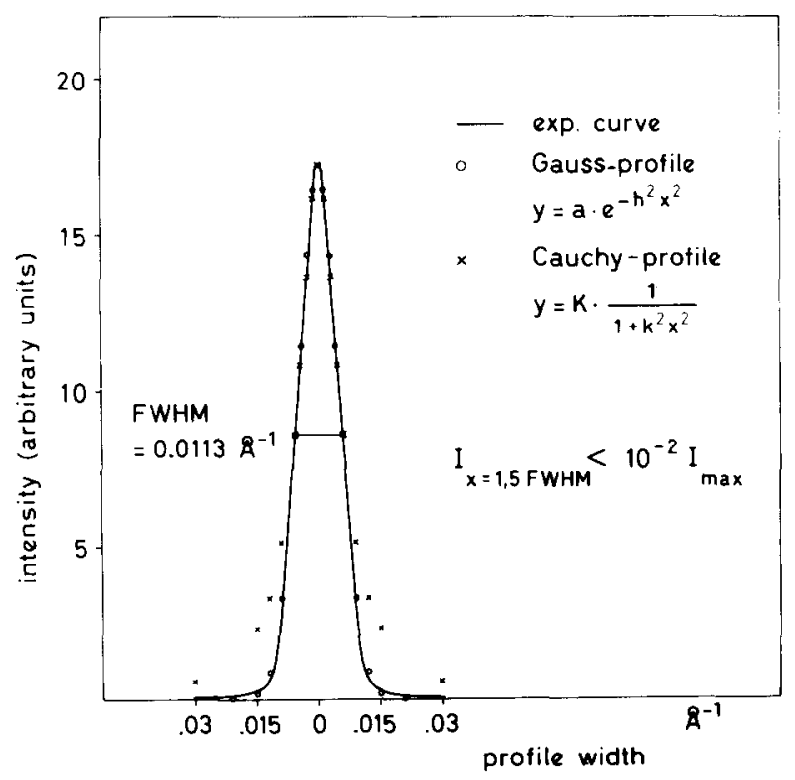

Fig. 9. Comparison of the instrumental profile with a Gauss and a Cauchy distribution. 
distribution of the specular beam. But the analysis of the interference profiles of $(h, k)$ reflexes needs a corrected width of the instrumental function with respect to the diffraction geometry.

For the specific diffraction conditions, such as electron energy $E_{\mathrm{p}}=68 \mathrm{eV}$ and an angle of incidence $\vartheta_{0}=47^{\circ}$, the divergence of the primary beam is known. Using the reciprocal lattice of the (101)Au surface (fig. 10) the divergence of the diffracted beams can be derived. With these results the calculation of the full width at half maximum (FWHM) of the instrumental function, which is necessary for the deconvolution of the $\left(0 \frac{1}{2}\right)$ and the $(01)$ beam profiles, can be done.

The upper part of the intensity distribution of the $\left(0 \frac{1}{2}\right)$ and $(01)$ reflexes (fig. 11) is well approximated by the Cauchy function

$y=K /\left(1+k^{2} x^{2}\right)$

only the asymmetric wings differ from the exact shape of the Cauchy curve.

In the intensity range above the FWHM the curves of the Gaussian and the Cauchy distributions show only small differences; this fact may be understood by the identical first terms of the corresponding expansion into a series. Only the parts of the intensity curves below the FWHM differ essentially.

Therefore, the shape of the instrumental profile above the FWHM can well be represented by a Cauchy curve. With this assumption the part above the FWHM of the unknown intensity distribution $f(x)$ of the deconvoluted profile can be replaced

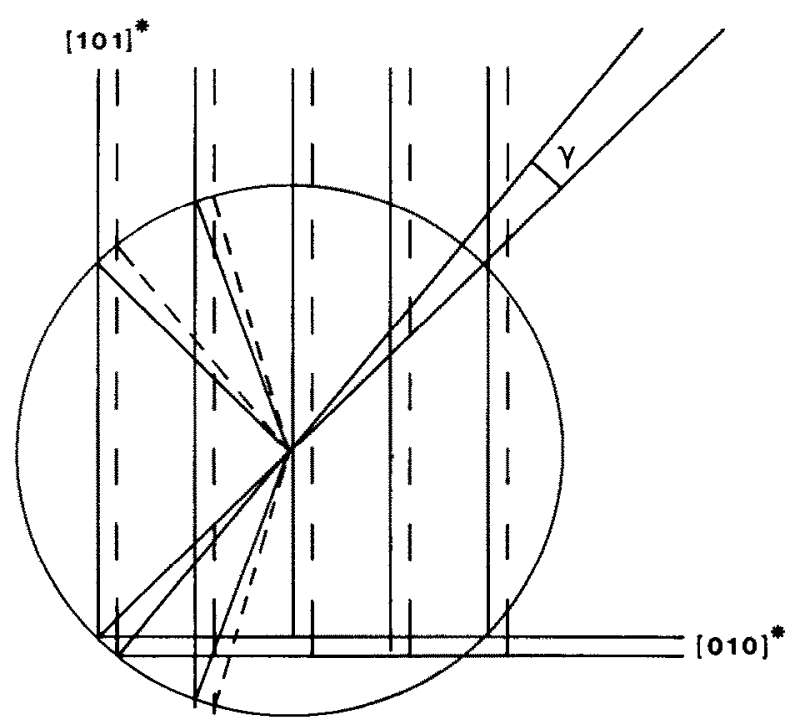

Fig. 10. Reciprocal lattice construction for the determination of the beam divergence as a function of of the diffraction angle. Ewald sphere for $E_{\mathrm{p}}=68 \mathrm{eV}$. Primary beam divergence $\gamma$. Angle of incidence of the primary beam $\vartheta_{0}=47^{\circ}$. 


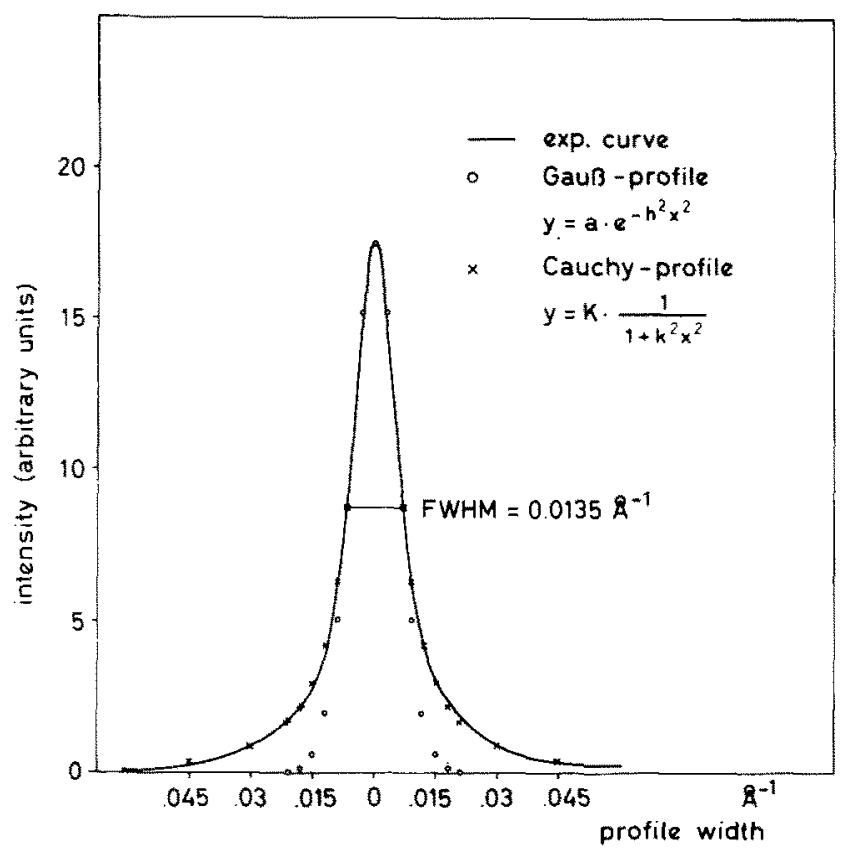

Fig. 11. Comparison of the intensity distribution of the 01 beam with a Gauss and Cauchy curve. Electron energy $E_{\mathrm{p}}=68 \mathrm{eV}$.

by a Cauchy profile; this procedure is allowed since the observed intensity curves of the reflexes correspond to Cauchy profiles.

The deconvolution procedure primarily influences the maximum and the central range of the beam profile; the intensity distribution of the lower part of the curve is not changed essentially. For this reason the deconvolution below the FWHM was completed by employing the remaining parts of the intensity profile. This approximate deconvolution was carried out practivally in the following way: First, the FWHM $b$ of the instrumental profile was subtracted from the FWHM B of the experimentally observed beam profile

$\beta=B-b$.

The new line width $\beta$ has been used to get the Cauchy curve

$f(x)=K /\left(1+k^{2} x^{2}\right), \quad k=1 / x_{\mathrm{FWHM}}=2 / \beta$,

which represents the shape of the deconvoluted intensity curve above the FWHM. The calibration of the factor $K$ with the maximum of the deconvoluted interference curve has been determined by the formula

$$
A_{\text {exp }}=\int_{-x_{\mathrm{FWHM}}}^{+x_{\mathrm{FWHM}}} f(x) \mathrm{d} x=K \int_{-x_{\mathrm{FWHM}}}^{+x_{\mathrm{FWHM}}} \frac{1}{1+k^{2} x^{2}} \mathrm{~d} x=\frac{\pi K}{2 k} .
$$


$A_{\text {exp }}$ is the area under the observed intensity profile within the limits $-x_{\text {FWHM }}$ and $+x_{\text {FWHM }}$. The shape of the unfolded curve below the FWHM is described by the corresponding part of the experimentally observed profile; by multiplication with the factor $K$ all functional values are approximated to the Cauchy curve. The individual values $k$ and $K$ of the (01) and the $\left(0 \frac{1}{2}\right)$ beams at different temperatures are given in table 1 .

This approximate deconvolution has been checked by an exact numerical deconvolution of some profiles. As the wings on both sides of the maximum of the experimental profile are asymmetric and differ from the exact shape of the Cauchy curve, the analytical expression is not suited to describe the interference function.

By means of a smoothing program, an intensity distribution of the interference has been calculated, using the intensity values determined from the shape of the experimental curve.

Table 1

Data for the deconvolution of the beam profiles

(a) 01 beam

\begin{tabular}{rlllll}
\hline \multicolumn{2}{l}{$\begin{array}{lll}\text { Temperature } \\
\left({ }^{\circ} \mathrm{C}\right)\end{array}$} & $\begin{array}{l}\text { FWHM } \beta \\
\left(\times 10^{-3} \AA\right)\end{array}$ & $\begin{array}{l}k \\
\left(\AA^{-1}\right)\end{array}$ & $\begin{array}{l}K \\
\left(\times 10^{16} \AA\right)\end{array}$ & $\begin{array}{l}A_{\exp } \\
\left(\times 10^{16} \AA^{2}\right)\end{array}$ \\
\hline 25 & 298 & 4.30 & 465.12 & 6218.15 & 21.00 \\
140 & 413 & 6.70 & 298.51 & 1947.87 & 10.25 \\
245 & 518 & 7.15 & 277.78 & 1166.39 & 6.55 \\
300 & 573 & 7.45 & 266.67 & 910.92 & 5.33 \\
400 & 678 & 8.80 & 227.27 & 528.11 & 3.65 \\
440 & 715 & 10.20 & 169.08 & 337.03 & 2.70 \\
500 & 773 & 11.40 & 175.44 & 210.03 & 1.80 \\
585 & 858 & 13.30 & 150.38 & 100.52 & 1.05 \\
\hline
\end{tabular}

(b) $0 \frac{1}{2}$ beam

\begin{tabular}{|c|c|c|c|c|c|}
\hline \multicolumn{2}{|c|}{ Temperature } & \multirow{2}{*}{$\begin{array}{l}\text { FWHM } \beta \\
\left(\times 10^{-3} A\right)\end{array}$} & \multirow{2}{*}{$\begin{array}{l}k \\
\left(\AA^{-1}\right)\end{array}$} & \multirow{2}{*}{$\begin{array}{l}K \\
\left(\times 10^{16} \AA\right)\end{array}$} & \multirow{2}{*}{$\begin{array}{l}A_{\exp } \\
\left(\times 10^{16} A^{2}\right)\end{array}$} \\
\hline$\left({ }^{\circ} \mathrm{C}\right)$ & $(\mathrm{K})$ & & & & \\
\hline 25 & 298 & 12.85 & 155.04 & 3269.80 & 33.00 \\
\hline 140 & 413 & 14.20 & 140.85 & 1470.50 & 16.40 \\
\hline 245 & 493 & 14.20 & 140.85 & 1143.23 & 12.75 \\
\hline 300 & 563 & 16.00 & 125.00 & 855.46 & 10.75 \\
\hline 400 & 673 & 20.05 & 99.50 & 288.94 & 4.55 \\
\hline 440 & 713 & 29.20 & 68.49 & 148.25 & 3.40 \\
\hline 480 & 753 & 49.00 & 40.82 & 68.86 & 2.65 \\
\hline 515 & 788 & 61.80 & 32.36 & 45.33 & 2.20 \\
\hline
\end{tabular}


The analytical expressions of the two Gaussion functions $y=\exp \left(-h^{2} x^{2}\right), \quad$ with $\left\{\begin{array}{l}h^{2}=0.21 \mathrm{~mm}^{-2} \text { for the }(01) \text { reflex }, \\ h^{2}=0.19 \mathrm{~mm}^{-2} \text { for the }\left(0 \frac{1}{2}\right) \text { reflex }\end{array}\right.$ can be used directly as apparative profile.
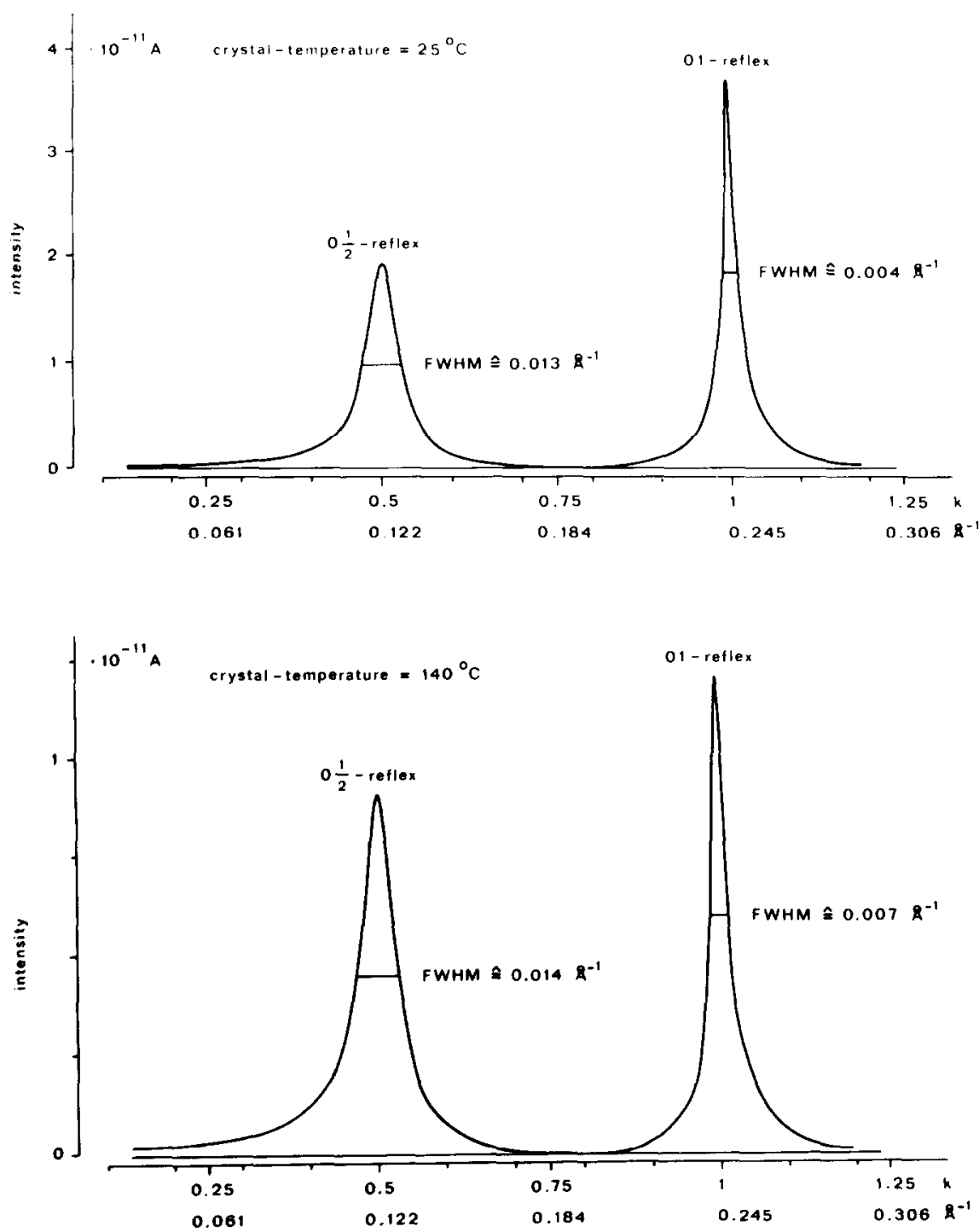

Fig. 12. Deconvoluted beam profiles at tempcratures of 25 and $140^{\circ} \mathrm{C}$. 
A determination of the intrinsic beam profile requires the solution of the equation

$h(x)=\int_{-\infty}^{+\infty} f(x) g(x-y) \mathrm{d} x$

where $g(x)$ represents the Gaussian apparative profile, $f(x)$ the interference profile and $h(x)$ the true intensity distribution.

With the Fourier transforms of $h(x)$ and $g(x)$ the expression for the intrinsic profile is given by

$f(x)=\frac{1}{(2 \pi)^{1 / 2}} \int_{-\infty}^{+\infty} \frac{H(y)}{G(y)} \mathrm{e}^{-2 \pi i x y} \mathrm{~d} y$

with

$H(y)=\frac{1}{(2 \pi)^{1 / 2}} \int_{-\infty}^{+\infty} h(x) \mathrm{e}^{2 \pi i x y} \mathrm{~d} x$ and $G(y)$ analogously.

After a series expansion of $H(y)$ and $G(y)$ the equation can be solved numerically. A comparison of profiles deconvoluted with both methods shows an agreement of the curves of better than $10 \%$.

Deconvoluted beam profiles - as demonstrated in the drawings of fig. 12 , representing two examples at crystal temperatures of 25 and $140^{\circ} \mathrm{C}-$ can be used to verify model structures by calculating suitable intensity distributions.

A structure discussion with a corresponding surface model will be shown in part IV.

\section{Summary}

LEED experiments on clean (101)Au surfaces show diffraction pattern with a characteristic $(1 \times 2)$ superstructure. The surface atoms are arranged in chains closely packed along the [101]] direction; the lattice periodicity in [010] is doubled. This atomic arrangement is stable up to a surface temperature of $400^{\circ} \mathrm{C}$; within the range of temperatures of 400 and $500^{\circ} \mathrm{C}$, a transformation in the $(1 \times 1)$ bulk-type lattice structure is observed. The distinct diffuseness of both, the (01) and the $\left(0 \frac{1}{2}\right)$ beams in the $[010]^{*}$ dircetion of the diffraction diagrams, can be attributed to a disorder of the superstructure periodicity. As broadening of the profile width appears only in one direction, a one-dimensional disorder problem becomes evident. Structure model discussions together with the disorder theory require the intrinsic interference profile. The deconvolution of the observed profiles, broadened by the instrumental function, is carried out with an approximation method. 


\section{Acknowledgement}

The authors wish to thank the Deutsche Forschunggemeinschaft (SFB 128) for financial support. We also gratefully acknowledge the technical assistance of $\mathrm{H}$. Plöckl and K.H. Wiechers.

\section{References}

[1] J.B. Pendry, Low Energy Electron Diffraction. (Academic Press, London, 1974).

[2] W. Moritz, H. Jagodzinski and D. Wolf, Surface Sci. 77 (1978) 249

H. Jagodzinski, W. Moritz and D. Wolf, Surface Sci. 77 (1978) 233

[3] D. Wolf, Thesis, Univ. München (1972);

H. Plöckl, K.H. Wiechers and D. Wolf, Vakuum Techn. 19 (1970) 110.

[4] N.J. Taylor, Rev, Sci, Instr. 40 (1969) 792.

[5] J.C. Tracy and G.K. Bohn, Rev. Sci. Instr. 41 (1970) 591.

[6] D. Wolf, to be published.

[7] This special construction is due to the 6 inch OD flanges with maximum 4 inch ID on the chamber. Now, in a diffraction chamber with larger dimensions, an improved 4-grid LEED system with $250 \mathrm{~mm}$ diam. and an angle of acceptance from the target at $120^{\circ}$ is used.

[8] D.G. Fedak and N.A. Gjostein, Surface Sci. 8 (1967) 77.

[9] P.W. Palmberg and T.N. Rhodin, Phys. Rev. 161 (1967) 586.

[10] Fink and Yates, Atomic Data 1 (1970) 385. 